\title{
SÍNTESE DE NANOPARTÍCULAS MAGNÉTICAS POR COMBUSTÃO UTILIZANDO RECIPIENTE DE FUNDO CÔNCAVO*
}

\author{
André Ben-Hur da Silva Figueiredo ${ }^{1}$ \\ Daniele Gomes Carvalho ${ }^{2}$ \\ Letícia dos Santos Aguilera ${ }^{3}$ \\ Ronaldo Sérgio de Biasit7
}

\section{Resumo}

Nanopartículas de $\mathrm{ZnFe}_{2} \mathrm{O}_{4}, \mathrm{CoFe}_{2} \mathrm{O}_{4}$ e $\mathrm{Fe}_{3} \mathrm{O}_{4}$ foram sintetizadas pelo método de combustão utilizando recipiente de fundo côncavo e comparadas as sintetizadas em recipiente de fundo plano. As amostras foram caracterizadas por difração de raios $\mathrm{X}$ (DRX), ressonância ferromagnética (RFM) e espectroscopia no infravermelho por transformada de Fourier (IV-TF). Os resultados mostraram que o método permite obter partículas de dimensões nanométricas e que as amostras sintetizadas no recipiente de fundo côncavo apresentam menor tamanho de cristalito. Também foi observado que as amostras de $\mathrm{CoFe}_{2} \mathrm{O}_{4}$ e $\mathrm{Fe}_{3} \mathrm{O}_{4}$ sintetizadas no recipiente côncavo têm menor quantidade de $\mathrm{CO}_{2}$ e $\mathrm{H}_{2} \mathrm{O}$ adsorvido.

Palavras-chave: Nanopartículas Magnéticas; Ferrita; Ondas de Combustão.

\section{COMBUSTION SYNTHESIS OF MAGNETIC NANOPARTICLES USING A CONCAVE CONTAINER}

\begin{abstract}
Nanoparticles of $\mathrm{ZnFe}_{2} \mathrm{O}_{4}, \mathrm{CoFe}_{2} \mathrm{O}_{4}$ and $\mathrm{Fe}_{3} \mathrm{O}_{4}$ were synthesized by the combustion method using a concave and a flat container. The samples were characterized by $\mathrm{X}$ ray diffraction (XRD), ferromagnetic resonance (FMR) and Fourier transform for infrared spectroscopy (FT-IR). The results show that the method yielded nanometersized particles, and that the samples synthesized in the concave container had parameter smaller crystallite size. It was also found that the samples of $\mathrm{Fe}_{3} \mathrm{O}_{4}$ and $\mathrm{CoFe}_{2} \mathrm{O}_{4}$ synthesized in the concave container had smaller amounts of adsorbed $\mathrm{CO}_{2}$ and $\mathrm{H}_{2} \mathrm{O}$.
\end{abstract}

Keywords: Magnetic Nanoparticles; Ferrite; Combustion Waves.

1 Bacharel em Física, Mestre em Engenharia Nuclear, Doutor em Ciência dos Materiais, Professor, Curso Básico e Seção de Engenharia Mecânica e de Materiais, Instituto Militar de Engenharia, Rio de Janeiro, RJ, Brasil.

2 Bacharel em Física, Mestra em Ciência dos Materiais, Doutora em Ciência dos Materiais, Seção de Engenharia Mecânica e de Materiais, Instituto Militar de Engenharia, Rio de Janeiro, RJ, Brasil.

3 Bacharel em Engenheira de Petróleo, Mestra em Ciência dos Materiais, Doutoranda em Ciência dos Materiais, Seção de Engenharia Mecânica e de Materiais, Instituto Militar de Engenharia, Rio de Janeiro, RJ, Brasil.

4 Bacharel em Engenharia Elétrica, Mestre em Engenharia Elétrica, Ph.D. em Engenharia Elétrica Professor Emérito Seção de Engenharia Mecânica e de Materiais, Instituto Militar de Engenharia, Rio de Janeiro, RJ, Brasil.. 


\section{INTRODUÇÃO}

O processo de síntese por combustão constitui a evolução de uma das principais técnicas da química úmida, denominada síntese por combustão em solução (SCS) [1,2]. O método foi proposto na literatura para a síntese de nanomateriais, por ser mais simples, rápido e econômico que os métodos tradicionais $[3,4,5]$ O método de ondas de combustão, mais sofisticado que o de combustão, mas que também permite uma síntese rápida e barata [6], consiste em promover uma ignição local da mistura reagente, seguida por uma onda de combustão que se propaga ao longo da amostra. Na onda de combustão é usualmente utilizada uma canaleta plana de alumina com o fundo homogeneamente aquecido por uma fonte de calor. A ignição se faz em um dos extremos, utilizando uma segunda fonte de calor [6,7]. Uma forma mais simples de produzir uma onda de combustão, que foi utilizada no presente trabalho, envolve o uso de um recipiente de queima com gradiente de temperatura, no qual a temperatura da extremidade mais quente é suficiente para promover a ignição.

\section{MATERIAIS E MÉTODOS}

Foram sintetizadas e caracterizadas seis amostras, rotuladas como $\mathrm{Zn} R \mathrm{RP}$, Zn_RC, Co_RP, Co_RC, Fe_RP e Co_RC. O rótulo foi formado com o símbolo do íon divalente e o tipo de recipiente; plano (RP) ou côncavo(RC).

\subsection{Materiais utilizados}

Nitratos $\mathrm{Zn}\left(\mathrm{NO}_{3}\right)_{2} .6 \mathrm{H}_{2} \mathrm{O}, \mathrm{Co}\left(\mathrm{NO}_{3}\right)_{2} .6 \mathrm{H}_{2} \mathrm{O}$ e $\mathrm{Fe}\left(\mathrm{NO}_{3}\right)_{3} .9 \mathrm{H}_{2} \mathrm{O}$, com $98 \%$ de pureza, da marca Aldrich, e glicina $\mathrm{C} 2 \mathrm{H} 5 \mathrm{NO} 2$, com98,5\% de pureza, da marca Aldrich, foram dissolvidos em água deionizada para obter três soluções precursoras.

\subsection{Preparação das amostras}

Cada solução foi dividida em partes iguais entre dois recipientes: uma placa de Petri de fundo plano e o um recipiente tipo vidro de relógio, ambos com $10 \mathrm{~cm}$ de diâmetro. Para cada recipiente a água excedente foi evaporada e em seguida foi feita a ignição, gerando respectivamente combustão homogênea e uma onda de combustão que se propagou ao longo do material. A glicina é o combustível da reação de combustão, sendo reduzida pelos íons dos nitratos. Obedecendo ao equilíbrio estequiométrico, a reação exotérmica pode ser expressa como (Equação 1): 
A temperatura de combustão foi controlada ajustando a razão entre a quantidade de glicina e a quantidade de nitrato $(\mathrm{G} / \mathrm{N})$ em 1,0.

\subsection{Caracterização}

O tamanho médio dos cristalitos foi determinado a partir dos difratogramas obtidos em um difratômetro de Raios X Expert Pro Panalytical com radiação Cu-Ka $(1,5418 \AA \hat{)})$, do Setor de Cristalografia e Raios X do Departamento de Física Aplicada do CBPF. Foi empregado para análise o software TOPAS que tem como base 0 método Rietveld e utiliza os Parâmetros Fundamentais de Perfil.

Os espectros de RFM das amostras foram obtidos à temperatura ambiente no Laboratório de Ressonância Magnética do Instituto Militar de Engenharia, em um espectrômetro Varian E-12. Os espectros foram registrados usando os seguintes parâmetros: frequência de micro-ondas, $9,5 \mathrm{GHz}$; frequência de modulação, 100 $\mathrm{kHz}$.

A caracterização dos grupos funcionais presentes nas amostras foi feita por espectroscopia no infravermelho por transformada de Fourier IV-FT, em um espectrômetro da marca Shimadzu modelo IR Prestige-21, no Laboratório de Espectroscopia da Seção de Engenharia Química do Instituto Militar de Engenharia IME. As análises foram realizadas na faixa de 300 a $4000 \mathrm{~cm}^{-1}$. As amostras foram analisadas por feixe transmitido, sendo que foi usada uma matriz de $\mathrm{KBr}$ na proporção de 100 para 1.

\section{RESULTADOS E DISCUSSÃO}

\subsection{Difratogramas de raios $X$}

Nos difratogramas das amostras de $\mathrm{ZnFe}_{2} \mathrm{O}_{4}, \mathrm{CoFe}_{2} \mathrm{O}_{4}$ e $\mathrm{Fe}_{3} \mathrm{O}_{4}$, mostrados na Figura 2, observa-se as linhas típicas da estrutura da ferrita, como em Yan et al.[1], Vaidyanathan et al.[8], Tronc et al. [9] e Kavas et al.[10] . A Tabela 2 apresenta para cada amostra: as fases formadas; tamanho médio de cristalito; parâmetro de rede; massa específica e o valor do parâmetro de ajuste GOF (goodness of fit). 

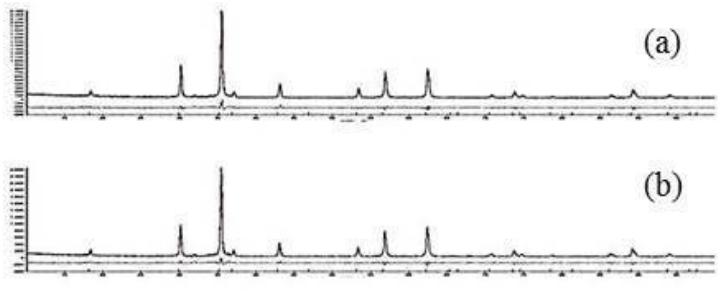

(c)

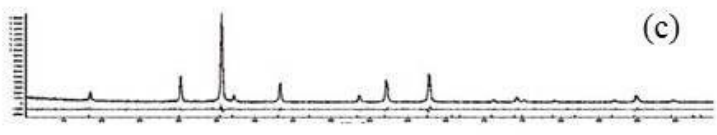

(d)

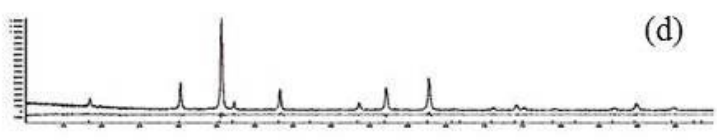

(e)
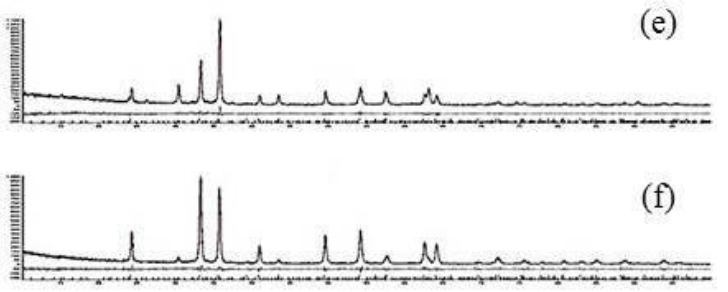

Figura 1: Difratogramas de raios $X$ das amostras: (a) Zn_RP; (b) Zn_RC; (c) Co_RP;(d) Co_RC; (e) $\mathrm{Fe} \_\mathrm{RP}$ e(f) Fe_RC.

Tabela1: Parâmetros obtidos a partir dos difratogramas de raios $\mathrm{X}$ das amostras de $\mathrm{ZnFe}_{2} \mathrm{O}_{4}$,

\begin{tabular}{|c|c|c|c|c|c|}
\hline AMOSTRA & $\begin{array}{c}\text { FASE } \\
\text { FORMADA }\end{array}$ & $\begin{array}{l}\text { TAMANHO } \\
\text { MÉDIO DE } \\
\text { CRISTALITO } \\
\text { (D) }(\mathrm{nm})\end{array}$ & $\begin{array}{l}\text { PARÂMETRO DE } \\
\text { REDE (A) }\end{array}$ & $\begin{array}{c}\text { MASSA } \\
\text { ESPECIFICA } \\
\text { (d) }\left(\mathrm{g} / \mathrm{cm}^{3}\right)\end{array}$ & GOF \\
\hline $\begin{array}{c}\mathrm{ZnFe}_{2} \mathrm{O}_{4} \\
\text { Plano }\end{array}$ & $100 \% \mathrm{ZnFe}_{2} \mathrm{O}_{4}$ & 63 & $\begin{array}{c}\text { Cúbica } \\
\text { a }-8,441\end{array}$ & 5,323 & 1,268 \\
\hline $\begin{array}{l}\mathrm{ZnFe}_{2} \mathrm{O}_{4} \\
\text { Côncavo }\end{array}$ & $100 \% \mathrm{ZnFe}_{4}$ & 57 & $\begin{array}{c}\text { Cúbica } \\
\text { a }-8,439\end{array}$ & 5,327 & 1,256 \\
\hline $\begin{array}{l}\mathrm{CoFe}_{2} \mathrm{O}_{4} \\
\text { Plano }\end{array}$ & $100 \% \mathrm{CoFe}_{2} \mathrm{O}_{4}$ & 59 & $\begin{array}{c}\text { Cúbica } \\
\text { a }-8,400\end{array}$ & 5,258 & 1,127 \\
\hline $\begin{array}{l}\mathrm{CoFe}_{2} \mathrm{O}_{4} \\
\text { Côncavo }\end{array}$ & $100 \% \mathrm{CoFe}_{2} \mathrm{O}_{4}$ & 54 & $\begin{array}{c}\text { Cúbica } \\
a-8,396\end{array}$ & 5,265 & 1,114 \\
\hline \multirow{2}{*}{$\begin{array}{l}\mathrm{Fe}_{3} \mathrm{O}_{4} \\
\text { Plano }\end{array}$} & $\begin{array}{c}54,54 \% \\
\text { Hematita }\end{array}$ & \multirow{2}{*}{54} & $\begin{array}{c}\text { Trigonal } \\
\mathrm{a}-5,037 \\
\mathrm{c}-13,757\end{array}$ & 5,262 & \multirow{2}{*}{1,175} \\
\hline & $\begin{array}{c}45,46 \% \\
\text { Maguemita }\end{array}$ & & $\begin{array}{c}\text { Tetragonal } \\
a-8,348 \\
c-25,077\end{array}$ & 4,779 & \\
\hline \multirow{2}{*}{$\begin{array}{l}\mathrm{Fe}_{3} \mathrm{O}_{4} \\
\text { Côncavo }\end{array}$} & $\begin{array}{l}90,64 \% \\
\text { Hematita }\end{array}$ & \multirow{2}{*}{52} & $\begin{array}{c}\text { Trigonal } \\
a-5,036 \\
c-13,753\end{array}$ & 5,266 & \multirow{2}{*}{1,234} \\
\hline & $\begin{array}{c}9,36 \% \\
\text { Maguemita }\end{array}$ & & $\begin{array}{c}\text { Tetragonal } \\
a-8,346 \\
c-25,034\end{array}$ & 4,790 & \\
\hline
\end{tabular}




\subsection{Espectros de Ressonância Ferromagnética}

A Figura 2 mostra os espectros de ressonância ferromagnética das amostras sintetizadas em um recipiente plano e em um recipiente côncavo.
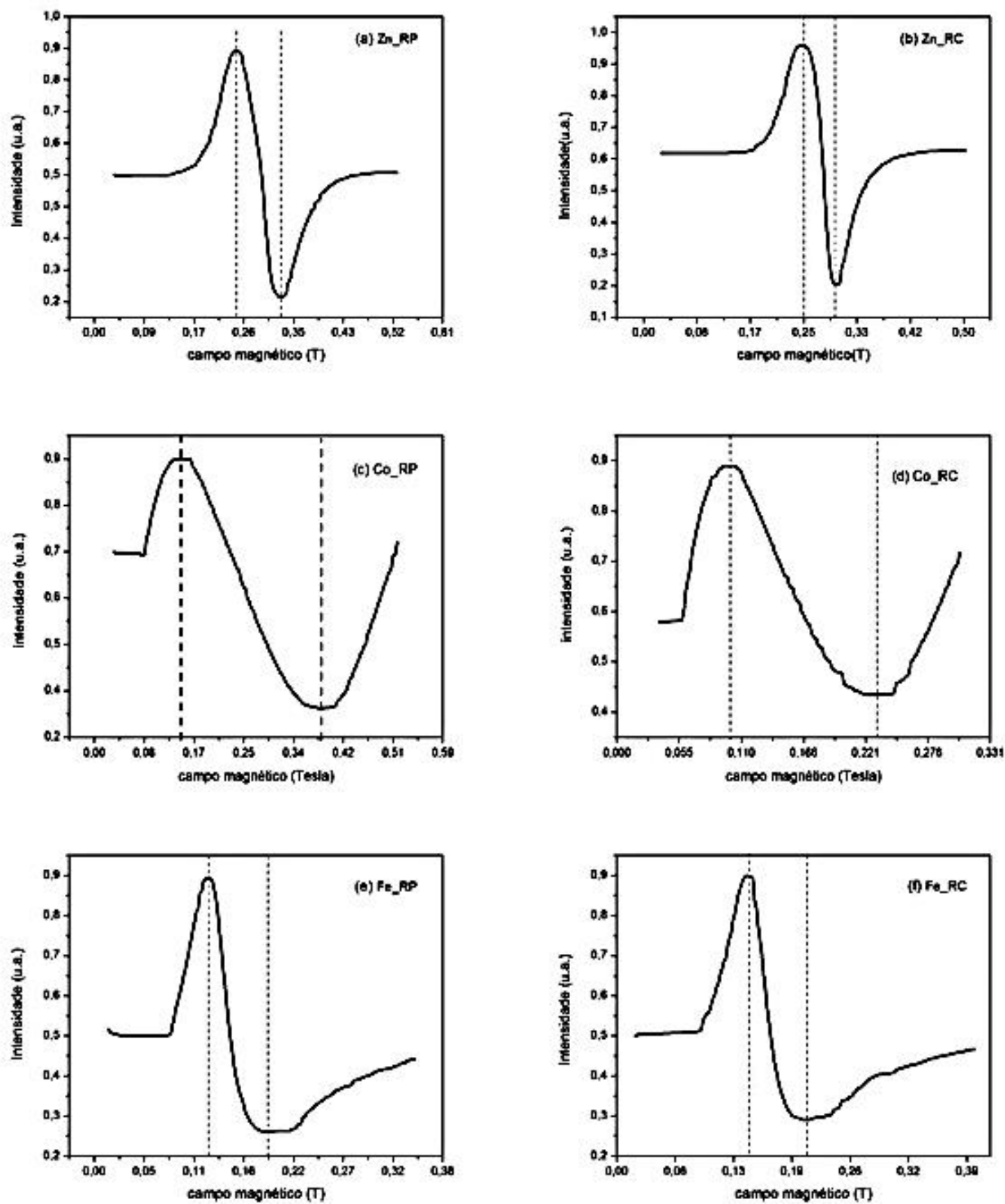

Figura 2: Espectros de RME das amostras: (a) Zn_RP; (b) Zn_RC; (c) Co_RP;(c) Co_RC; (d) Fe_RP e(e) Fe RC.

A Tabela 2 apresenta as larguras de linha pico a pico das amostras sintetizadas em um recipiente plano e em um recipiente côncavo. 
Tabela2 Largura de linha pico-a-pico das amostras de $\mathrm{ZnFe}_{2} \mathrm{O}_{4}, \mathrm{CoFe}_{2} \mathrm{O}_{4}$ e $\mathrm{Fe}_{3} \mathrm{O}_{4}$.

\begin{tabular}{c|c|c|c}
\hline AMOSTRA & $\begin{array}{c}\Delta H_{\text {PP }}(\mathrm{mT}) \\
\text { PLANO }\end{array}$ & $\begin{array}{c}\Delta H_{P P}(\mathrm{mT}) \\
\text { CÓNCAVO }\end{array}$ & $\begin{array}{c}\text { VARIAÇ̊̃o } \\
\%\end{array}$ \\
\hline $\mathrm{ZnFe}_{2} \mathrm{O}_{4}$ & 62 & 44 & 29,0 \\
\hline $\mathrm{CoFe}_{2} \mathrm{O}_{4}$ & 457 & 419 & 8,3 \\
\hline $\mathrm{Fe}_{3} \mathrm{O}_{4}$ & 218 & 206 & 5,5 \\
\hline
\end{tabular}

\subsection{Espectro no Infravermelho por Transformada de Fourier}

A Figura 3 apresenta os espectros de transmissão no infravermelho por transformada de Fourier das amostras sintetizadas: (a) em um recipiente côncavo como fabricadas $(\mathrm{RC})$; (b) em um recipiente plano com posterior tratamento térmico a $700^{\circ} \mathrm{C}$ por $30 \mathrm{~min}$. $[R P(700)]$ e (c) em um recipiente plano como fabricadas $(\mathrm{RC})$.
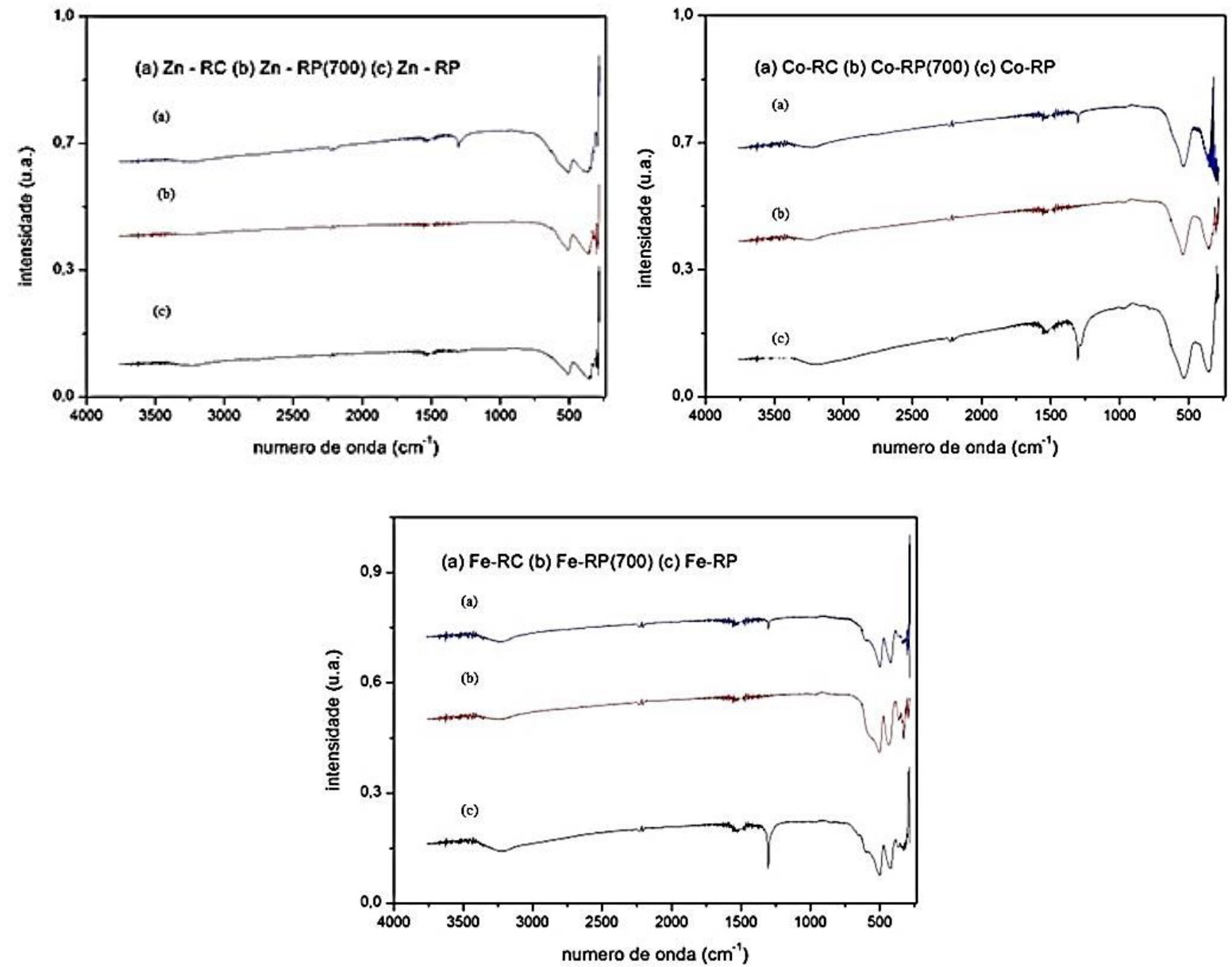

Figura 3: Espectro de transmissão no infravermelho de amostras sintetizadas: (a) em um recipiente côncavo como fabricadas (RC); (b) em um recipiente plano com posterior tratamento térmico a $700^{\circ} \mathrm{C}$ por 30 min. $[R P(700)]$ e (c) em um recipiente plano como fabricadas $(R C)$.

A Figura 4 apresenta os espectros de transmissão no infravermelho de amostras sintetizadas (a) em um recipiente côncavo como fabricadas (RC); (b) em um recipiente plano como fabricadas $(\mathrm{RP})$. 

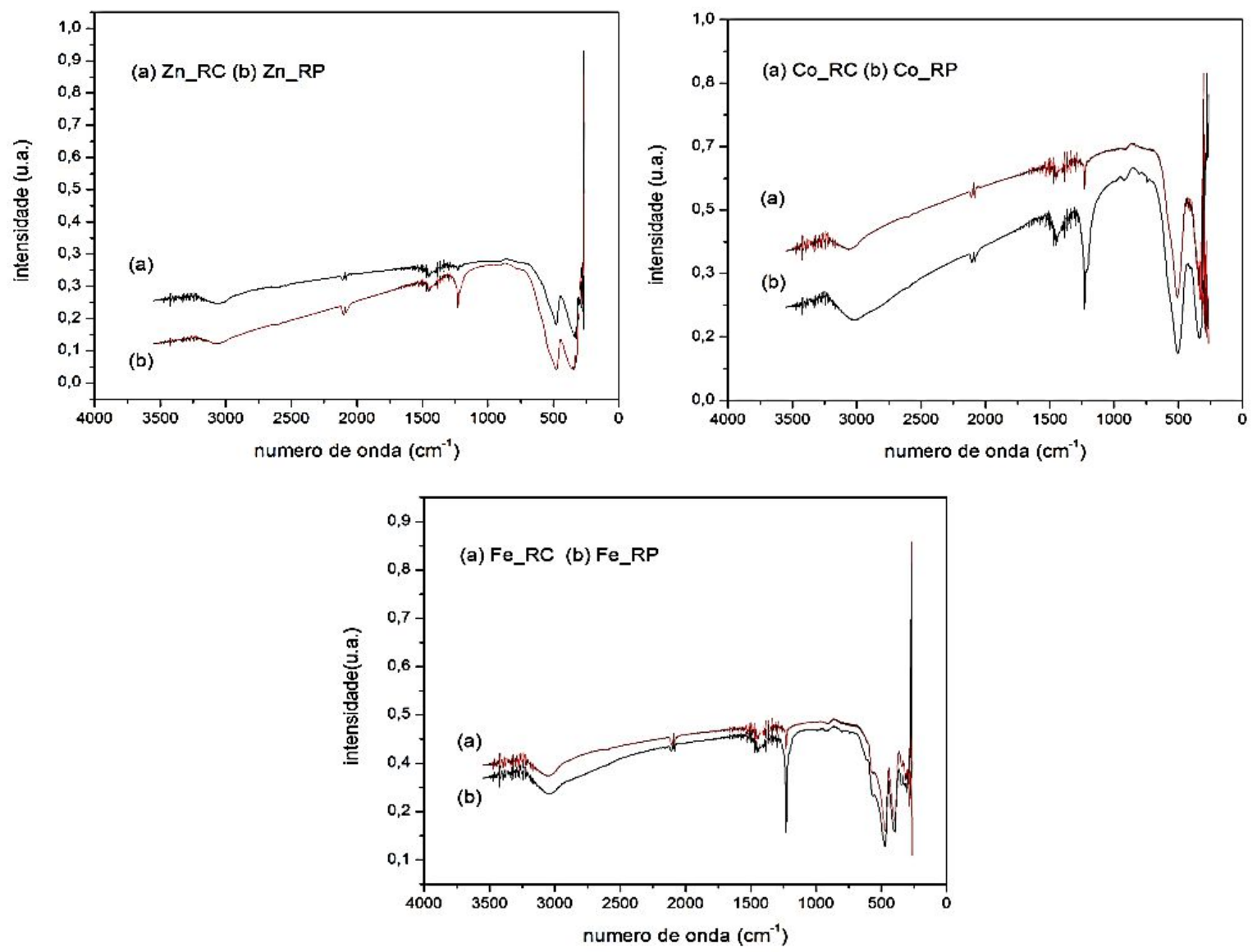

Figura 4: Espectro de transmissão no infravermelho de amostras sintetizadas no recipiente: (a) em um recipiente côncavo como fabricadas $(\mathrm{RC})$ e (b) em um recipiente plano como fabricadas (RP).

\subsection{DISCUSSÃO}

A Tabela 1 mostra os resultados obtidos a partir dos difratogramas, usando $o$ método de Rietveld com um nível de refinamento aceitável, indicado por um GOF (goodness of fit) entre 1,000 e 1,500. Os resultados mostram que todas as amostras como fabricadas podem ser consideradas nanométricas, uma vez que o tamanho médio dos cristalitos é menor que $63 \mathrm{~nm}$. A massa específica das amostras sintetizadas no recipiente côncavo é maior do que as sintetizadas no recipiente plano, enquanto o parâmetro de rede é menor. Na Tabela 2, as larguras de linha pico a pico, $\Delta H_{p p}$, das amostras sintetizadas em um recipiente plano são maiores que as das amostras sintetizadas em um recipiente côncavo, o que sugere que a forma do recipiente de queima afeta a anisotropia magnética dos três materiais.

$\mathrm{Na}$ Figura 3, a faixa de absorção compreendida entre 3000 a $3400 \mathrm{~cm}^{-1}$ sugere estiramentos $\mathrm{O}-\mathrm{H}$ dos nitratos, glicina e de moléculas de água presentes na solução reagente e fixados por ligações de hidrogênio à superfície das amostras $[8,9,10]$. Os picos em 1382,93 a $1388,75 \mathrm{~cm}^{-1}$ sugerem vibrações de estiramento do grupo funcional carboxila $\left(-\mathrm{CO}_{2}\right)$ e os picos entre $500 \mathrm{a} 600 \mathrm{~cm}^{-1}$ estão associados a íons metálicos em sítios tetraédricos, enquanto os picos entre 350 a $450 \mathrm{~cm}^{-1}$ estão associados a íons metálicos em sítios octaédricos [11,12]. Nas três amostras, é possível observar que tanto os picos na faixa de 3000 a $3400 \mathrm{~cm}^{-1}$ como na faixa 
entre 1382,93 e $1388,75 \mathrm{~cm}^{-1}$ foram reduzidos com o tratamento térmico a $700^{\circ} \mathrm{C}$ por $30 \mathrm{~min}$.

Na Figura 4, também é possível observar que as amostras sintetizadas no recipiente côncavo possuem menor volume de água e carboxila adsorvida na superfície das nanopartículas, que as amostras sintetizadas no recipiente plano.

\section{CONCLUSÃO}

Os resultados obtidos neste trabalho mostram que a nova rota no método de ondas de combustão utilizando um recipiente côncavo permite sintetizar nanopartículas de $\mathrm{ZnFe}_{2} \mathrm{O}_{4}, \mathrm{CoFe}_{2} \mathrm{O}_{4}$ e $\mathrm{Fe}_{3} \mathrm{O}_{4}$ com tamanho de cristalito menor que no método de combustão usando recipiente plano. O menor tamanho $(52 \mathrm{~nm})$ foi os das partículas de $\mathrm{Fe}_{3} \mathrm{O}_{4}$. Os resultados mostram também que a síntese no recipiente côncavo reduz a anisotropia magnética das partículas quando comparadas as fabricadas no recipiente plano. Por fim, no recipiente côncavo há menos água e carboxila adsorvida na superfície das nanopartículas de $\mathrm{CoFe}_{2} \mathrm{O}_{4}$ e $\mathrm{Fe}_{3} \mathrm{O}_{4}$.

Suspeita-se que o menor tamanho de cristalito esteja relacionado a formação de ondas de combustão gerado pela orientação da queima da amostra [6]. Para o recipiente côncavo, a orientação foi da borda para o centro, seguido de menor intervalo de tempo de queima em cada região da amostra, durante a combustão. Como no recipiente plano não houve orientação, é possível ter aumentado o intervalo de queima devido ao comportamento aleatório da combustão. Também foi observado menor retenção de água durante a combustão no recipiente côncavo. 0 motivo é que grande parte da água adsorvida surge de oxigênio na atmosfera. Como a experiência foi realizada com volumes iguais, a geometria côncava tem menor superfície de exposição com a atmosfera que a superfície plana. Devido a isto, menor será a água adsorvida na superfície das nanopartículas, menor será as nanopartículas e menor será a anisotropia magnetocristalina.

\section{Agradecimentos}

Os autores agradecem à FAPERJ, à CAPES e ao CNPq pelo apoio financeiro.

\section{REFERÊNCIAS}

[1] YAN, C. -H., XU, Z. -G., CHENG, F.-X., WANG, Z.-M., SUN, L.-D., LIAO, C.-S, JIA, J.-T., "Nanophased $\mathrm{CoFe}_{2} \mathrm{O}_{4}$ prepared by combustion method", Solid State Commun., v. 111, p. 287, 1999.

[2] BURGOS-MONTES, O., MORENO, R., COLOMER, M.T., FARIÑAS, J.C., "Influence of combustion synthesis of mullite powers", J. Euro. Cer. Soc., v. 26, pp. 3365-3372, 2006.

[3] KIMINAMI, R.H.G.A., FOLZ., D. C., CLARCK, D. E., "Microwave synthesis of alumina powders", Am. Ceram. Soc. Bull. v. 79, n. 3, p. 63-67, 2000.

[4] MIMANI, T.,PATIL, K.C., "Solution combustion synthesis of nanoscale oxides and their composites", Mater. Phys. Mech., v. 4, pp. 134-137,2001. 
[5] CARVALHO, D. G., FIGUEIREDO, A.B.S., "Síntese e caracterização de nanopartículas de $\mathrm{NiFe}_{2} \mathrm{O}_{4}$ utilizando o método de sol-gel/combustão e combustão homogênea", Revista Matéria, v. 18, n. 2, pp. 1323-1328, 2013.

[6] DE BIASI, R.S., FIGUEIREDO, A.B.S., FERNANDES, A.A.R. e LARICA, C., "Synthesis of cobalt ferritenanoparticles using combustion waves", Solid State Commun., v. 144, p. 15, 2007.

[7] FIGUEIREDO, A.B.S., Estudo comparativo dos métodos de combustão e sol-gel para obtenção de nanopartículas de $\mathrm{CoFe}_{2} \mathrm{O}_{4}$, Tese de D.Sc., IME, Rio de Janeiro, RJ, Brasil, 2007.

[8] VAIDYANATHAN, G., SENDHILNATHAN, S., "Characterization of $\mathrm{Co}_{-x} \mathrm{Zn}_{x} \mathrm{Fe}_{2} \mathrm{O}_{4}$ nanoparticles synthesized by co-precipitation method", J. Magn. Magn.Mater.,v. 403, p. 2157, 2008.

[9] TRONC, E., EZZIR, A., CHERKAOUI, R., CHANÉAC, C., NOGUĖS, M., KACHKACHI, H., FIORANI,D., TESTA, A.M., GRENĖCHE, JOLIVET J.P., "Surface-related properties of $\gamma$ $\mathrm{Fe}_{2} \mathrm{O}_{3}$ nanoparticles", J.Magn. Magn.Mater.,v. 221, p. 63-79, 2000.

[10] KAVAS, H., KASAPOGLU, N., BAYKAL, A., KÖSEOGLU, Y., "Characterization of $\mathrm{NiFe}_{2} \mathrm{O}_{4}$ nanoparticles synthesized by various methods", Chemical Papers, v. 62 p. 450-455, 2009.

[11] HUANG, Y., TANG, Y., WANG, J., CHEN, Q., "Synthesis of $\mathrm{MgFe}_{2} \mathrm{O}_{4}$ nanocrystallites under mild conditions", Mater.Chem. Phys., pp. 394-397, v. 97, 2006.

[12] WALDRON, R. D.,“Infrared Spectra of Ferrites”, Physical Review, pp. 1727-1735, v. 99, n. 6, 1955. 